\title{
Numerical models of isolated rotating compact stars
}

\author{
J. Novak*, M. Oertel, D. Chatterjee and A. Sourie \\ LUTH, Observatoire de Paris, PSL Research University, CNRS, \\ Université Paris Diderot, Sorbonne Paris Cité, 5 place Jules Janssen, 92195 Meudon \\ E-mail: jerome.novak@obspm. fr
}

\begin{abstract}
Realistic global neutron star models require numerical approach. Here, we briefly motivate and recall the general-relativistic framework for the description of rotating compact stars and show how global observable quantities can be computed unambiguously. Then, we present some recent results on the building of more detailed physical models, including realistic microphysics, following two directions. First, we describe the inclusion of magnetic field and its effects on both, global equilibrium and equation of state, and finally we discuss superfluid (two-fluid) models in an attempt to model glitch phenomena.
\end{abstract}

The Modern Physics of Compact Stars 2015

30 September 2015 - 3 October 2015

Yerevan, Armenia

${ }^{*}$ Speaker. 


\section{Introduction}

The building of compact stars models requires inputs from many different fields of physics, with overall conditions that can hardly be tested on Earth. These include nuclear physics, with cold, highly asymmetric nuclear matter; general relativity, with stars possessing a very strong gravitational field (only a black hole can possess a stronger one); electromagnetism, with an intense magnetic field up to about $10^{13} \mathrm{~T}$; and special relativity, with rotation velocities approaching that of light for rapidly rotating stars. Thus, the combination of equations arising from these fields makes it often necessary to use numerical simulations for the setting of a global theoretical model.

First, in order to motivate the use of general relativity (GR), we here give a qualitative difference between neutron star models in Newtonian theory and general relativistic ones: the existence of a maximal mass. Indeed, if one solves for spherically symmetric configurations with increasing central density as a parameter in Newtonian theory of gravity, mass can increase to arbitrary large values. On the contrary, if one uses instead GR, there is a critical density for which the mass reaches a maximal value and above which it decreases. A measure of the influence of GR on a self-gravitating object of mass $M$ and radius $R$ is the so-called compactness ratio, defined as

$$
\mathscr{C}=G M / R c^{2},
$$

with $G$ the gravitational constant and $c$ the speed of light. The highest value is $\mathscr{C}=0.5$ for a black hole, while $\mathscr{C} \simeq 0.2$ for compact stars, and $\mathscr{C} \sim 10^{-6}$ for our Sun.

The simplest model in GR is obtained when assuming spherical symmetry, i.e. static equilibrium, together with the fact that matter is made of a cold perfect fluid, at $\beta$-equilibrium. The structure of such a compact star can be described by the well-known Tolman - Oppenheimer Volkoff (TOV) system [1, 2], coupled to an equation of state (EoS). The line elements, giving the GR metric reads:

$$
d s^{2}=-e^{2 v(r)} c^{2} d t^{2}+\left(1-\frac{2 G m(r)}{r c^{2}}\right)^{-1} d r^{2}+r^{2}\left(d \theta^{2}+\sin ^{2} \theta d \varphi^{2}\right) ;
$$

with $m(r)$ and $v(r)$ are the two gravitational potentials. Matter is described by the pressure $p(r)$ and energy density $\varepsilon(r)$ profiles. TOV equations are then a system of coupled ordinary differential equations in terms of the coordinate radius $r$ :

$$
\begin{aligned}
& \frac{d m}{d r}=4 \pi r^{2} \frac{\varepsilon(r)}{c^{2}} \\
& \frac{d v}{d r}=\left(1-\frac{2 G m(r)}{r c^{2}}\right)^{-1}\left(\frac{G m(r) c^{2}}{r^{2}}+4 \pi G p(r)\right), \\
& \frac{d p}{d r}=-[\varepsilon(r)+p(r)] \frac{d \nu}{d r}
\end{aligned}
$$

Each model can be parameterized by a value of the central of pressure (or, equivalently, density) and varying this value, one gets a sequence of equilibrium configurations, which can be displayed as a curve in the mass-radius plane for a given EoS. Each EoS gives a priori a different mass-radius relation, see Fig. 1. This figure also shows the influence of rotation, by displaying mass-radius diagrams for rotating sequences at $700 \mathrm{~Hz}$, which is about the fastest known pulsar. 
The difference between the radius measured at the pole or at the equator is noticeable, too, as it can reach up to $1 \mathrm{~km}$ for a $1.4 M_{\odot}$ star. Therefore, one must be careful when defining radii for compact stars: if rotation is not taken into account, important errors can be made.

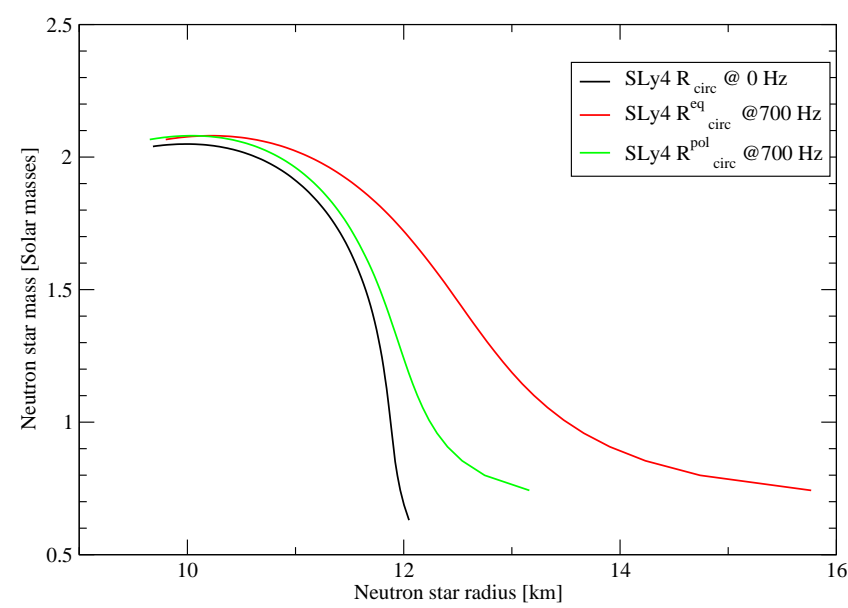

Figure 1: Mass radius relations for static and rotating compact stars, using the EoS SLy4 by Douchin \& Haensel [3]. For the rotating case, stars are rotating at $700 \mathrm{~Hz}$, which is about the fastest rotating pulsar observed. In the rotating case, both curves for polar $R^{\mathrm{pol}}$ and equatorial $R^{\mathrm{eq}}$ radii are given.

The TOV system has been devised in the 30's, and is quite easy to solve numerically. It is a very interesting approach to test rapidly an EoS or some new idea in microphysics. However, rotating compact star models now have a long history, too and there exist several easy-to-use tools to compute such models. The first and oldest one is the perturbative approach by Hartle and Thorne (1968) [4], called slow rotation approximation. The starting point is the solution of the TOV system upon which a rotating solution is given in powers of the angular velocity, supposed to be small. The first numerical model of rotating relativistic stars has been obtained by Bonazolla and Maschio (1971) [5], using the so-called Lewis-Papapetrou coordinates. A year later, Wilson [6] extended the model to the case of differentially rotating stars. Then, Friedmann et al. (1986) [7] and Lattimer et al. (1990) [8] incorporated realistic EoSs for rapidly rotating neutron star models. Bocquet et al. (1995) [9] were the first to include magnetic field, thus solving coupled EinsteinMaxwell equations. Furthermore, there now exist several numerical codes solving the rotating relativistic star structure, some of them being publicly available: the KEH solver by Komatsu et al. (1989) [10], rostar from the LORENE library, based on the approach by Bonazzola et al. (1993) [11], the widely used rns code by Stergioulas and Friedmann (1995) [12]. These three numerical approaches have been compared by Nozawa et al. (1998) [13], showing excellent agreement. More recently, new codes have emerged, in particular, the very accurate one by Ansorg et al. (2002) [14], able to describe strongly deformed stars and XNS, handling magnetic field too, by Pili et al. (2014) [15]. Here, we shall give some details about rotating relativistic star models in Sec. 2, before showing two lines of improvement of the models: the inclusion of magnetic field, with Einstein-Maxwell equations and a magnetic field dependent EoS in Sec. 3; and the two-fluid 
approach, to describe superfluid phenomena such as pulsar glitches in Sec. 4.

\section{Stationary and axisymmetric approach}

As we plan to describe rotating stars in GR, spacetime is assumed to possess some symmetries, which are described in GR by the existence of Killing vector fields. Thus, we suppose that there exist two Killing vector fields: a timelike one to account for stationarity, and a spacelike one, with closed orbits, for axisymmetry. Under such conditions, it is possible to choose adapted coordinates, such that the metric depends only on two coordinates $(r, \theta)$ and takes the form (quasi-isotropic gauge) (see e.g. [11]):

$$
d s^{2}=-N^{2} d t^{2}+A^{2}\left(d r^{2}+r^{2} d \theta^{2}\right)+B^{2} r^{2} \sin ^{2} \theta(d \varphi-\omega d t)^{2},
$$

with the additional requirement of circularity for the matter, which implies the absence of meridional currents and no mixed toroidal/poloidal magnetic field. Note that this gauge is quite different from the one used for the TOV system (1.3) in spherical symmetry.

In this gauge, the Einstein equation for a star rigidly rotating at the frequency $\Omega$ turn into a system of four coupled non-linear elliptic partial differential equations [11]:

$$
\begin{aligned}
& \Delta_{3} v=4 \pi A^{2}\left(E+3 p+(E+p) U^{2}\right)+\frac{B^{2} r^{2} \sin ^{2} \theta}{2 N^{2}}(\partial \omega)^{2}-\partial v \partial(v+\beta) \\
& \tilde{\Delta}_{3}(\omega r \sin \theta)=-16 \pi \frac{N A^{2}}{B}(E+p) U-r \sin \theta \partial \omega \partial(3 \beta-v) \\
& \Delta_{2}[(N B-1) r \sin \theta]=16 \pi N A^{2} B p r \sin \theta \\
& \Delta_{2}(v+\alpha)=8 \pi A^{2}\left[p+(E+p) U^{2}\right]+\frac{3 B^{2} r^{2} \sin ^{2} \theta}{4 N^{2}}(\partial \omega)^{2}-(\partial v)^{2}
\end{aligned}
$$

with the following notations $v:=\ln N, \alpha:=\ln A, \beta:=\ln B$ and

$$
\begin{aligned}
\Delta_{2} & :=\frac{\partial^{2}}{\partial r^{2}}+\frac{1}{r} \frac{\partial}{\partial r}+\frac{1}{r^{2}} \frac{\partial^{2}}{\partial \theta^{2}} \\
\Delta_{3} & :=\frac{\partial^{2}}{\partial r^{2}}+\frac{2}{r} \frac{\partial}{\partial r}+\frac{1}{r^{2}} \frac{\partial^{2}}{\partial \theta^{2}}+\frac{1}{r^{2} \tan \theta} \frac{\partial}{\partial \theta} \\
\tilde{\Delta}_{3} & :=\Delta_{3}-\frac{1}{r^{2} \sin ^{2} \theta} \\
\partial a \partial b & :=\frac{\partial a}{\partial r} \frac{\partial b}{\partial r}+\frac{1}{r^{2}} \frac{\partial a}{\partial \theta} \frac{\partial b}{\partial \theta} .
\end{aligned}
$$

In Eqs. (2.2)-(2.5), $U$ and $E$ are respectively the fluid 3-velocity and energy density, both as measured by the locally non-rotating observer: $U=B r \sin \theta(\Omega-\omega) / N, E=\Gamma^{2}(\varepsilon+p)-p$ is the velocity in the $\varphi$-direction, $\Gamma=\left(1-U^{2}\right)^{-1 / 2}$ the Lorentz factor. Note that sources of Eqs. (2.2)(2.5) contain non-linear metric terms of non-compact support. So, contrary to spherical symmetry there is no matching to any known vacuum solution at the surface of the star (no Birkhoff theorem), and boundary conditions for the metric system can only be imposed at $r \rightarrow \infty$.

The matter source terms come from the model of perfect fluid for the energy-momentum tensor:

$$
T^{\mu v}=(\varepsilon+p) u^{\mu} u^{v}+p g^{\mu v}
$$


where $u^{\mu}$ is the fluid 4-velocity, $p$ its pressure and $\varepsilon$ its total energy density. This simple matter model can be extended in the following directions:

- superfluidity can be taken into account, under the two-fluid approach as shown in Sec. 4 ,

- electromagnetic field in a perfect conductor with magnetization, see Sec. 3, or in a superconductor (Bonazzola and Gourgoulhon 1996 [16]),

- a fluid crust with EoS-based model (as e.g. SLy4 by Douchin and Haensel 2001 [3]),

- an elastic crust, with a solid-type model (Carter and Quintana 1972 [17], Beig and Schmidt 2003 [18], Gundlach et al. 2012 [19], ...).

The equilibrium of this perfect fluid can be described in the stationary, axisymmetric and circular case, by the conservation of the energy-momentum tensor, which turns into a simple first integral of motion [11]:

$$
H+v-\ln \Gamma=\text { const, }
$$

with the log-enthalpy

$$
H=\ln \left(\frac{\varepsilon+p}{n_{B} c^{2}}\right)
$$

and $n_{B}$ the baryon density. As in the TOV case, the system is closed by the description of microscopic properties of the fluid through the $\operatorname{EoS} p(H), \varepsilon(H)$.

In order to extract from the numerical results observationally relevant information, it is necessary in GR to define gauge independent quantities. The gravitational mass is the mass felt by a particle orbiting around the star; in our case it can be equivalently computed either as the Komar mass (from stationarity property), or as the ADM mass (asymptotic flatness of spacetime). The baryon mass is just the total number of baryons in the star, multiplied by the baryon mean mass. The circumferential radius is computed integrating the line element $d s$ defined by Eq. (2.1) over a star circumference at the equator or passing through the poles. In our case of axisymmetry, it is possible to define a total angular momentum, and to compute it from the asymptotic behavior of the metric potential $\omega$. Formulas and more details can be found e.g. in Bonazzola et al. (1993) [11].

\section{Magnetic field}

Compact stars are known to possess strong magnetic field from pulsar observations. Indeed, their magnetic field can be estimated by the accelerated dipole model to be in the range $10^{4} \rightarrow 10^{8} \mathrm{~T}$ for most of pulsars ${ }^{1}$. However, it is known that very highly magnetized neutron stars exist, which are called magnetars (Duncan and Thompson 1992 [20]). They are observed as anomalous X-ray pulsars (AXP) or soft gamma-ray repeaters (SGR), they show a low rotation period $P \sim 1-10 \mathrm{~s}$ and high deceleration $\dot{P}$. With the magnetar model, their dipolar magnetic field is estimated at the surface up to $10^{10}-10^{11} \mathrm{~T}$. Such a magnetic field should have an influence on the global structure of the compact star.

\footnotetext{
${ }^{1} 1 \mathrm{~T}=10^{4} \mathrm{G}$
} 
This question has been addressed mainly in two ways: either focusing on microphysics or on global structure. The first approach consists in looking at the effect of magnetic field on the EoS and uses rather simple global model, with the TOV system, assuming spherical symmetry, which is not compatible with the fact that there are no magnetic monopoles. However, using additional assumptions on the structure of the energy-momentum tensor (e.g. the existence of parallel and perpendicular pressures) several authors have studied magnetic-field dependent EoSs [21, 22, 23, 24]. On the other hand, global models have been computed in axisymmetry, including Lorentz force and consistent magnetic pressure $[9,25,26,27,28]$. In these studies, very simple EoSs have been used, mostly polytropic, which did not take into account the magnetic field. Hereafter, we shall present a numerical model that combines both approaches (see also Chatterjee et al. 2015 [29]).

The starting point is a microscopic Lagrangian where the density functions of fermions $\psi$ are coupled to an electromagnetic field $F_{\mu \nu}$ :

$$
\mathscr{L}=-\bar{\psi}(x)\left(D_{\mu} \gamma^{\mu}+m\right) \psi(x)-\frac{1}{4 \mu_{0}} F_{\mu v} F^{\mu \nu} .
$$

From this one gets the microscopic energy-momentum tensor:

$$
T^{\mu v}=-\frac{1}{\mu_{0}} F^{\mu \alpha} F_{\alpha}^{v}+\frac{1}{2} \bar{\psi}\left(\gamma^{\mu} D^{v}+\gamma^{v} D^{\mu}\right) \psi+g^{\mu v} \mathscr{L},
$$

which, after a thermal average, gives the macroscopic energy-momentum tensor, which is used in the global model:

$$
\left\langle T^{\mu v}\right\rangle=(\varepsilon+p) u^{\mu} u^{v}+p g^{\mu v}+\frac{1}{2}\left(F_{\tau}^{v} M^{\tau \mu}+F_{\tau}^{\mu} M^{\tau v}\right)-\frac{1}{\mu_{0}}\left(F^{\mu \alpha} F_{\alpha}^{v}+\frac{g^{\mu v}}{4} F_{\alpha \beta} F^{\alpha \beta}\right),
$$

with $M_{\mu \nu}$ the magnetization tensor in presence of $b^{\mu}$, the magnetic field in the fluid rest frame:

$$
M_{\mu v}=\frac{x}{\mu_{0}} \varepsilon_{\alpha \beta \mu v} u^{\beta} b^{\alpha} .
$$

The model, which details are presented in Chatterjee et al. [29] is thus quite similar to that presented in Bocquet et al. [9], but with the equations containing new terms depending on the magnetization $x$ defined in Eq. (3.4). In particular, the model solves coupled Einstein-Maxwell equations with all fields depending only on $(r, \theta)$ coordinates, as in Sec. 2. Moreover, only poloidal magnetic field is considered, and the magnetic moment is aligned with the rotation axis. Free currents $j_{\text {free }}^{\sigma}$ decoupled from inert mass movement are sources of Maxwell equations, with additional terms from magnetization $x$ :

$$
\nabla_{\mu} F^{\sigma \mu}=\frac{1}{1-x}\left(\mu_{0} j_{\text {free }}^{\sigma}+F^{\sigma \mu} \nabla_{\mu} x\right)
$$

The microphysics calculations take into account the magnetic field in the fluid rest frame $b$ and, within our model the EoS provides pressure $p(H, b)$, energy density $\varepsilon(H, b)$ and magnetization $x(H, b)$ as functions of both enthalpy and magnetic field.

From the expression of the energy-momentum tensor (3.3), the equilibrium equation for matter (2.11) in presence of electromagnetic field reads:

$$
\nabla_{\mu} T^{\mu v}=\nabla_{\mu} T_{f}^{\mu v}-F^{v \mu} j_{\mu}^{\text {free }}-\frac{x}{2 \mu_{0}} F_{\sigma \tau} \nabla^{v} F^{\sigma \tau}
$$


where $T_{f}^{\alpha \beta}$ is the part of the energy-momentum tensor associated with a perfect fluid (2.10). This expression can be made more explicit:

$$
(\varepsilon+p)\left(\frac{1}{\varepsilon+p} \frac{\partial p}{\partial x^{i}}+\frac{\partial v}{\partial x^{i}}-\frac{\partial \ln \Gamma}{\partial x^{i}}\right)-F_{i \rho} j_{\text {free }}^{\rho}-\frac{x}{\mu_{0}} b \frac{\partial b}{\partial x^{i}}=0 .
$$

However, as shown by Chatterjee et al. (2015) [29], the last term containing the magnetization $x$ cancels when one writes that pressure $p$ depends on the magnetic field $b$, too. Thus the term

$$
\frac{\partial p}{\partial x^{i}}=\frac{\partial p}{\partial H} \frac{\partial H}{\partial x^{i}}+\frac{\partial p}{\partial b} \frac{\partial b}{\partial x^{i}}
$$

the first term giving the usual gradient of enthalpy and the second canceling out with the last term in Eq. (3.7). It means that equilibrium depends only on enthalpy gradient, together with gravitational, centrifugal and Lorentz forces, as for the case without magnetization (Cf. e.g. Bocquet et al. 1995 [9]). In particular, this means that there should be no assumption about the existence of parallel or perpendicular pressure.

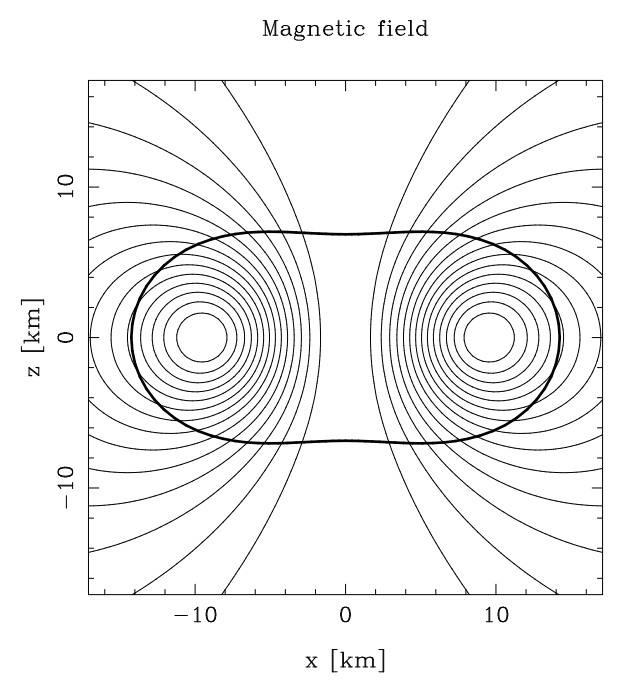

Figure 2: Magnetic field for a non-rotating star. The gravitational mass is $2.2 M_{\odot}$ and the magnetic field at the pole $8.2 \times 10^{13} \mathrm{~T}$. Thick line indicates the star's surface.

As an application, we have implemented the EoS for quark matter in MCFL phase by Noronha and Shovkovy (2008) [30], which consists in massless three-flavor MIT bag model (with a bag constant of $60 \mathrm{MeV} / \mathrm{fm}^{3}$ ), together with a NJL-type pairing interaction. As expected, we observe de Haas van Alphen oscillations, but the maximal magnetization $x \lesssim 10^{-3}$, which means that the overall effect on compact star structure should be small.

As an illustration, Fig. 2 shows the magnetic field resulting from our simulation for a nonrotating star, with a magnetic field-dependent EoS and magnetization taken into account. The deformation is due to the Lorentz force, which "pinches" the star along the magnetic axes. This figure shows the limits of our code, too, as it cannot give accurate results when the star becomes too strongly distorted in a torus-like shape (see also Cardall et al. 2001 [25]). This occurs when 


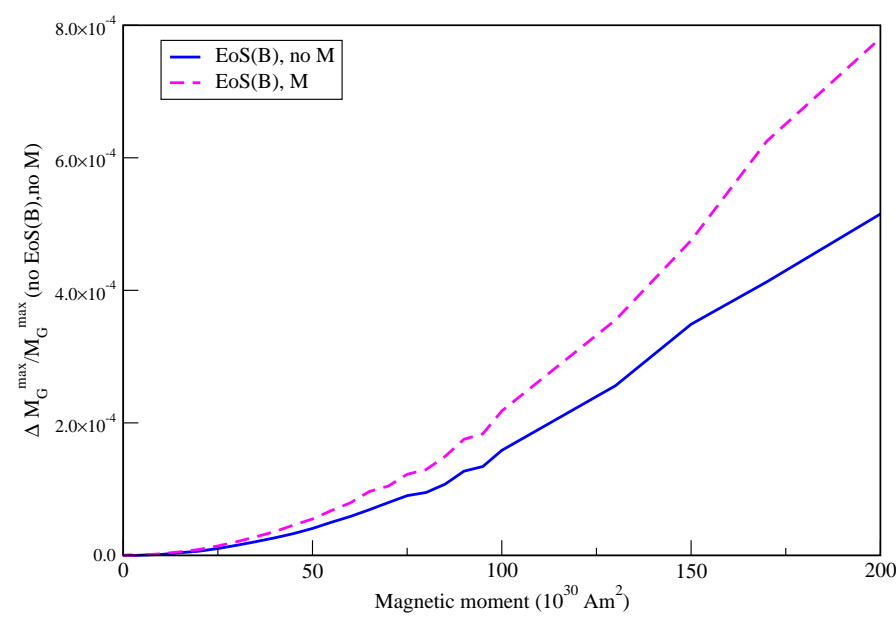

Figure 3: Relative influence on the maximal mass, as a function of magnetic moment of the star.

the magnetic pressure at the center becomes equivalent to the fluid one. Fig. 3 shows the relative difference in the computation of the maximal mass of a non-rotating compact star as a function of the magnetic moment, when taking into account the magnetization, the magnetic field-dependent EoS or none of them. From this figure, one can say that the influence of magnetization (if $x \neq 0$ ), or of the magnetic field in the EoS are very small (less than $10^{-3}$ ), within the case we have considered. This may be due to the very specific EoS we have chosen and, perhaps, some hadronic-type EoS would give higher numbers. However, it is to be noted that the very high magnetic moments used here are much higher than the strongest observed magnetars.

\section{Two-fluid models}

In this section, we do not consider magnetic field, but superfluid properties of neutron stars. The observations of pulsar glitches, or sudden spin-up, with long relaxation time-scales have given evidence for the existence of a superfluid component in neutron stars [31]. As a consequence, there should be several dynamically distinct components inside neutron stars. We here consider two fluids: the first one is composed of all charged particles, locked together via electromagnetic interactions on very short timescales. This fluid shall be generically called "protons", with an angular velocity $\Omega_{p}=\Omega$ the pulsar observed frequency. The second fluid is composed of superfluid neutrons with a slightly higher angular velocity $\Omega_{n} \gtrsim \Omega_{p}$.

The evolution of $\Omega_{n}$ and $\Omega_{p}$ are sketched in Fig. 4: with the loss of energy due to pulsar emission, $\Omega_{p}$ decreases, while $\Omega_{n}$ remains almost constant. At a critical lag between both velocities, the two components lock together, neutrons giving part of their angular momentum to protons, which are spun up (the glitch). After some time, the proton fluid relaxes and start slowing down again. There are two sorts of coupling between both fluids occurring in this scenario: a non-dissipative one, called entrainment, linked with strong interaction between nucleons, and a dissipative one, called mutual friction, linked with pinning and unpinning of superfluid vortices to/from the neutron 


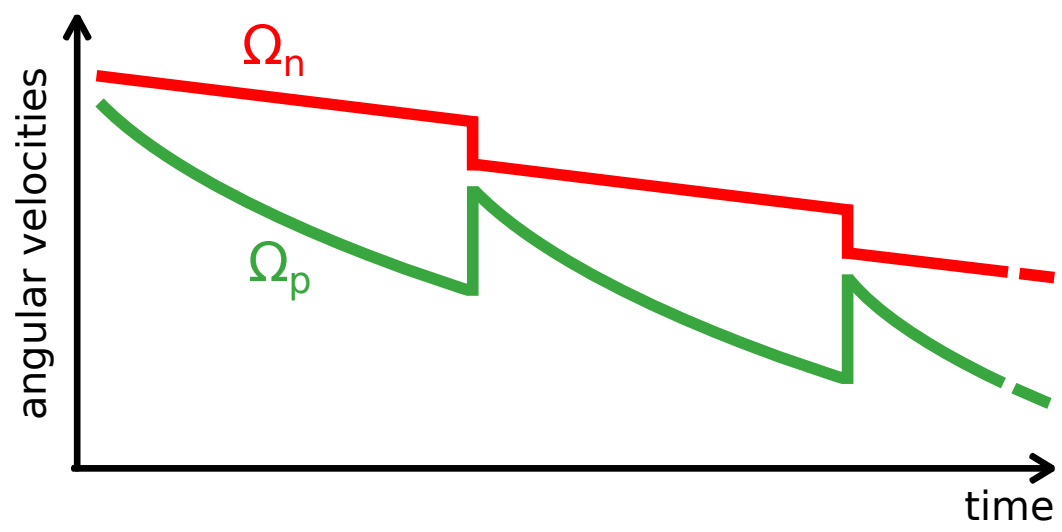

Figure 4: Schematic evolution of the two-fluid angular velocities during glitch phenomena.

star crust. Here, we show preliminary results from the numerical computations of equilibrium configurations of rotating superfluid neutron stars, considering two-fluid EoSs based on microphysics. We apply then these configurations to a simple numerical model for pulsar glitches.

To start with, we defined the energy-momentum tensor in the two-fluid case:

$$
T_{\alpha \beta}=n_{n \alpha} p_{\beta}^{n}+n_{p \alpha} p_{\beta}^{p}+\Psi g_{\alpha \beta},
$$

where $\Psi$ is the generalized pressure, $n_{n}^{\alpha}$ and $n_{p}^{\alpha}$ being respectively the 4-currents of neutrons and protons, $p_{\alpha}^{n}$ and $p_{\alpha}^{p}$, the corresponding conjugate 4-momenta. Indeed, without entrainment 4momenta are proportional to 4-velocities and therefore, to 4-currents, too. However, here we have (see e.g. Prix et al. 2005 [32]) that momenta are linear combinations of currents, using the entrainment matrix $\mathscr{K}^{X Y}$ :

$$
\begin{aligned}
p_{\mu}^{n} & =\mathscr{K}^{n n} n_{n \mu}+\mathscr{K}^{n p} n_{p \mu}, \\
p_{\mu}^{p} & =\mathscr{K}^{p n} n_{n \mu}+\mathscr{K}^{p p} n_{p \mu} .
\end{aligned}
$$

Numerical models with two fluids in rotating compact stars in GR were developed by Prix et al. (2005) [32], using a similar approach to that of Sec. 2, but with a non-realistic EoS.

We have developed a new EoS based on microphysics. In the case of two fluids and entrainment the EoS is giving e.g. energy density $\mathscr{E}$ as a function of neutron and proton densities $\left(n_{n}, n_{p}\right)$ and the relative speed between both fluids $\Delta$. Equivalently, using the first law of thermodynamics

$$
d \mathscr{E}=\mu^{n} d n_{n}+\mu^{p} d n_{p}+\alpha d \Delta^{2}
$$

the EoS can provide generalized pressure as a function of both chemical potentials and the relative speed:

$$
\Psi\left(\mu^{n}, \mu^{p}, \Delta^{2}\right) .
$$

The underlying microscopic model is based of relativistic mean field theory, where nucleon-nucleon interactions are modeled by the exchange of effective mesons $\sigma, \omega, \rho$ and $\delta$. We have built two such EoSs: DDH (without the $\delta$ meson) and DDH $\delta$ (with $\delta$ meson). Both were adapted to a two-fluid system coupled by entrainment following previous approaches by Comer and Joynt (2003) [33], or Gusakov et al. (2009) [34]. 
In the two-fluid model, glitches start when the difference in angular velocities is greater than some threshold $\Delta \Omega=\Omega_{n}-\Omega_{p} \gtrsim \Delta \Omega_{c}$. There are very few observational constraints concerning the rising of the frequency (see Fig. 4). In particular, the rising time $\tau_{r}$ has been constrained to $\tau_{r} \lesssim 40 \mathrm{~s}$ - 2 min for the Vela pulsar [35], and about a few hours for the Crab pulsar [36]. On the other hand, the hydrodynamic time scale, which is the typical time scale for the equilibrium equation (2.11), is of the order of $0.1 \mathrm{~ms}$ [37]. As this is much shorter than $\tau_{r}$, we can model the glitch rise by a series of equilibrium configurations taken from the numerical library LORENE (see Prix et al. 2005 [32]), which has been updated in order to be able to use the realistic EoSs described here above. As these EoSs cannot be computed within the rotating star equilibrium code, they are saved as tables, which are then read and interpolated by the code. The series of equilibrium models are taken at constant total baryon number and angular momentum (i.e. we neglect during the rise time the energy loss by pulsar emission).

Following the study in Newtonian framework by Sidery et al. (2010) [38], the evolution equations for both angular velocities as functions of time $\Omega_{n}(t)$ and $\Omega_{p}(t)$ are given by the evolution of each specie's angular momentum $\left(J_{n}, J_{p}\right)$ :

$$
\left\{\begin{array}{l}
\dot{J}_{n}=+\Gamma_{\mathrm{int}} \\
\dot{J}_{p}=-\Gamma_{\mathrm{int}}
\end{array}\right.
$$

Here, $\Gamma_{\text {int }}$ is the mutual friction moment (see also Langlois et al. 1998 [39] and Sidery et al. 2010 [38] for details):

$$
\Gamma_{\text {int }}=\mathscr{B}\left(\Omega_{p}-\Omega_{n}\right) \int \Gamma_{n} n_{n} \varpi_{n} h_{\perp}^{2} d^{3} \Sigma .
$$

In this equation, beside the neutron fluid density $n_{n}$ and Lorentz factor $\Gamma_{n}, \varpi_{n}$ is the macroscopic superfluid vorticity, $h_{\perp}^{2}$ is a geometric coefficient, $d^{3} \Sigma=A^{2} B r^{2} \sin \theta d r d \theta d \varphi$ is the volume element and $\mathscr{B}$ the mutual friction parameter.

As preliminary results, we show a sequence of equilibrium models, starting from an initial relative difference in angular velocities (glitch amplitude) of $10^{-6}$ in Fig. 5. As expected, the angular velocity of protons increases, whereas that of neutron decreases. Angular momentum is transferred from the fluid of neutrons to that of protons. Both fluids tend to a common angular velocity. Typical rise time can be deduced from a fit of these curves by an exponential function:

$$
\Omega_{n}(t)-\Omega_{p}(t) \propto e^{-t / \tau_{r}} .
$$

In the case displayed in Fig. 5, one finds $\tau_{r} \sim 8.5 \mathrm{~s}$, which is compatible with observations. Note however that this rising time is quite sensitive to the value of $\mathscr{B}$ in Eq. (4.6). For the case here, we have taken $\mathscr{B}=10^{-6}$, but some better resolved observations of pulsar glitches should give us more information about this mostly unknown variable.

\section{Conclusion}

We have presented here several models of rotating compact stars. There is a need for such models, where rotation is taken into account, in particular if one is discussing or studying radii of compact stars. Moreover, when looking at the magnetic field in such stars, it is absolutely 


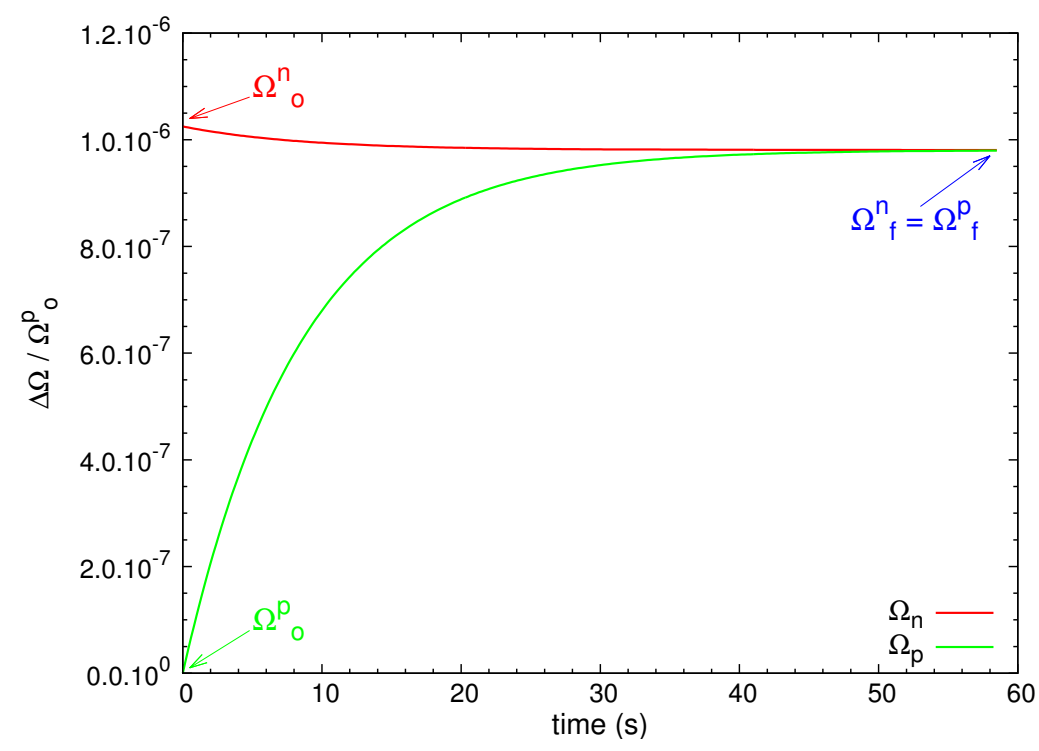

Figure 5: Numerical integration of the two fluid angular velocities relative differences as functions of time. The plotted quantities are $\left(\Omega_{n}(t)-\Omega_{p}(0)\right) / \Omega_{p}(0)$ (red) and $\left(\Omega_{p}(t)-\Omega_{p}(0)\right) / \Omega_{p}(0)$ (green). The star is rotating at $400 \mathrm{~Hz}$, with a total baryon mass of $1.7 M_{\odot}$.

compulsory to go beyond spherically symmetric models. There are now several numerical codes publicly available to study rotating or magnetic stars in GR. These codes have been extensively tested and are now fast, running on a simple laptop. With such codes, taken from the LORENE library, we have studied effect of magnetic field onto the EoS of compact stars, as well as that of magnetization (derivative of pressure with respect to magnetic field) on the global structure. The latter effect is very small, because there is a cancellation of terms depending on magnetization in the equilibrium equation for the star. Another study is dealing with two-fluid configurations of neutron stars, which are very interesting to model the glitch phenomenon in pulsars. In particular, we are able to recover the glitch rise time, which seems reasonable, having in mind the very few observational constraints.

The rotating compact star models shown here can be improved in many important ways. First, our glitch model needs to be improved and a parametric study must be undertaken, in particular concerning the EoS. Next, rotating star models in GR still lack of a good implementation of a solid crust. This is quite important because many observational neutron star properties depend on crust features. In particular, glitch models include pinning and unpinning of superfluid vortices in the crust. Therefore, more detailed models are expected here, as well as e.g. crust thermal properties. Temperature effects have numerous observational consequences, for instance for the cooling of (proto)-neutron stars. Thus, rotating models including temperature effects of out of $\beta$-equilibrium EoSs should be built, too.

\section{References}

[1] R.C Tolman, Static Solutions of Einstein's Field Equations for Spheres of Fluid, Phys. Rev. 55 (1939) 364. 
[2] J.R. Oppenheimer and G.M. Volkoff, On Massive Neutron Cores, Phys. Rev. 55 (1939) 374.

[3] F. Douchin and P. Haensel, A unified equation of state of dense matter and neutron star structure, Astron. Astrophys. 380 (2001) 151 [astro-ph/ 0111092 ].

[4] J.B. Hartle and K.S. Thorne, Slowly Rotating Relativistic Stars. II. Models for Neutron Stars and Supermassive Stars, Astrophys. J. 153 (1968) 807.

[5] S. Bonazzola and G. Maschio, Models of Rotating Neutron Stars in General Relativity, Proc. IAU Symp. 46 (1971) 346.

[6] J.R. Wilson, Models of Differentially Rotating Stars, Astrophys. J. 176 (1972) 195.

[7] J.L. Friedman, J.R. Ipser and L. Parker, Rapidly rotating neutron star models, Astrophys. J. 304 (1986) 115.

[8] J.M. Lattimer, M. Prakash, D. Masak and A. Yahil, Rapidly rotating pulsars and the equation of state, Astrophys. J. 355 (1990) L241.

[9] M. Bocquet, S. Bonazzola, E. Gourgoulhon and J. Novak, Rotating neutron star models with a magnetic field, Astron. Astrophys. 301 (1995) 757 [gr-qc/ 9503044 ].

[10] H. Komatsu, Y. Eriguchi and I. Hachisu, Rapidly rotating general relativistic stars. I - Numerical method and its application to uniformly rotating polytropes, Month. Not. Roy. Astron. Soc. 237 (1989) 355.

[11] S. Bonazzola, E. Gourgoulhon, M. Salgado and J.-A. Marck, Axisymmetric rotating relativistic bodies: A new numerical approach for "exact” solutions, Astron. Astrophys. 278 (1993) 421.

[12] N. Stergioulas and J. Friedmann, Comparing models of rapidly rotating relativistic stars constructed by two numerical methods, Astrophys. J. 444 (1995) 306 [astro-ph/9411032].

[13] T. Nozawa, N. Stergioulas, E. Gourgoulhon and Y. Eriguchi, Construction of highly accurate models of rotating neutron stars - comparison of three different numerical schemes, Astron. Astrophys. Suppl. 132 (1998) 331 [gr-qc/9804048].

[14] M. Ansorg, A. Kleinwächter and R. Meinel, Highly accurate calculation of rotating neutron stars, Astron. Astrophys. 381 (2002) L49 [astro-ph/0111080].

[15] A.G. Pili, N. Bucciantini and L. Del Zanna, Axisymmetric equilibrium models for magnetized neutron stars in General Relativity under the Conformally Flat Condition, Month. Not. Roy. Astron. Soc. 439 (2014) 3541 [1401.4308].

[16] S. Bonazzola and E. Gourgoulhon, Gravitational waves from pulsars: emission by the magnetic-field-induced distortion, Astron. Astrophys. 312 (1996) 675 [astro-ph/9602107].

[17] B. Carter and H. Quintana, Foundations of General Relativistic High-Pressure Elasticity Theory, Proc. Roy. Soc. London A 331 (1972) 57.

[18] R. Beig and B.G. Schmidt, Relativistic elasticity, Class. Quantum Grav. 20 (2003) 889 [gr-qc/0211054].

[19] C. Gundlach, I. Hawke and S.J. Erickson, A conservation law formulation of nonlinear elasticity in general relativity, Class. Quantum Grav. 29 (2012) 015005 [1107. 2551].

[20] R.C. Duncan and C. Thompson, Formation of very strongly magnetized neutron stars - Implications for gamma-ray bursts, Astrophys. J. 392 (1992) L9. 
[21] L. Paulucci, E.J. Ferrer, V. de La Incera and J.E. Horvath, Equation of state for the magnetic-color-flavor-locked phase and its implications for compact star models, Phys. Rev. D 83 (2011) 043009 [1010.3041].

[22] A. Rabhi, H. Pais, P.K. Panda and C. Providência, Quark-hadron phase transition in a neutron star under strong magnetic fields, J. Phys. G 36 (2009) 115204 [0909. 1114].

[23] V. Dexheimer, D.P. Menezes and M. Strickland, The influence of strong magnetic fields on proto-quark stars, J. Phys. G. 41 (2014) 015203 [1210 . 4526].

[24] R.Casali, L.B. Castro and D.P. Menezes, Hadronic and hybrid stars subject to density-dependent magnetic fields, Phys. Rev. C 89 (2014) 015805 [1307. 2651].

[25] C.Y. Cardall, M. Prakash and J.M. Lattimer, Effects of Strong Magnetic Fields on Neutron Star Structure, Astrophys. J. 554 (2001) 322 [astro-ph/ 0011148 ].

[26] K. Kiuchi and K. Kotake, Equilibrium configurations of strongly magnetized neutron stars with realistic equations of state, Month. Not. Roy. Astron. Soc. 385 (2008) 1327 [0 708 . 3597].

[27] J. Frieben and L. Rezzolla, Equilibrium models of relativistic stars with a toroidal magnetic field, Month. Not. Roy. Astron. Soc. 427 (2012) 3406 [1207 . 4035].

[28] N. Yasutake, K.Kiuchi and K. Kotake, Relativistic hybrid stars with super-strong toroidal magnetic fields: an evolutionary track with QCD phase transition, Month. Not. Roy. Astron. Soc. 401 (2010) 2101 [0910.0327].

[29] D. Chatterjee, T. Elghozi, J. Novak and M. Oertel, Consistent neutron star models with magnetic-field-dependent equations of state, Month. Not. Roy. Astron. Soc. 447 (2015) 3785 [1410.6332].

[30] J.L. Noronha and I.A. Shovkovy, Color-flavor locked superconductor in a magnetic field, Phys. Rev. D 76 (2007) 105030 [0708.0307].

[31] P.W. Anderson and N. Itoh, Pulsar glitches and restlessness as a hard superfluidity phenomenon, Nature 256 (1975) 25.

[32] R. Prix, J.Novak and G.L. Comer, Relativistic numerical models for stationary superfluid neutron stars, Phys. Rev. D 71 (2005) 043005 [gr-qc/ 0410023 ].

[33] G.L. Comer and R. Joynt, Relativistic mean field model for entrainment in general relativistic superfluid neutron stars, Phys. Rev. D 68 (2003) 023002 [gr-qc/ 0212083 ].

[34] M.E. Gusakov, E. Kantor and P. Haensel, Relativistic entrainment matrix of a superfluid nucleon-hyperon mixture: The zero temperature limit, Phys. Rev. C 80 (2009) 015803 [0907 . 0010 ].

[35] R.G. Dodson, P.M. McCulloch and D.R. Lewis, High Time Resolution Observations of the January 2000 Glitch in the Vela Pulsar, Astrophys. J. Lett. 564 (2002) L85 [astro-ph/ 0201005 ].

[36] A.G. Lyne, F.G. Smith and R.S. Pritchard, Spin-up and recovery in the 1989 glitch of the Crab pulsar, Nature 359 (1992) 706.

[37] S.L. Shapiro, Differential Rotation in Neutron Stars: Magnetic Braking and Viscous Damping, Astrophys. J. 544 (2000) 397 [astro-ph/ 0010493 ].

[38] T. Sidery, A. Passamonti and N. Andersson, The dynamics of pulsar glitches: contrasting phenomenology with numerical evolutions, Month. Not. Roy. Astron. Soc. 405 (2010) 1061 [0910.3918].

[39] D. Langlois, D.M. Sedrakian and B. Carter, Differential rotation of relativistic superfluid in neutron stars, Month. Not. Roy. Astron. Soc. 297 (1998) 1189 [astro-ph/9711042]. 